\title{
Strahlungs-induzierter subakut kutaner Lupus erythematodes oder außergewöhnliche Radiodermatitis?
}

\author{
Radiation-Induced Subacute Cutaneous Lupus Erythematosus or Extraordinary Radiodermatitis?
}

Autoren

Institute
R. Renner ${ }^{1}$, M. Sticherling ${ }^{2}$

Klinik für Dermatologie, Venerologie und Allergologie, Universität Leipzig A. ö.R.

Hautklinik, Universitätsklinikum Erlangen

\section{Bibliografie}

Dol http://dx.doi.org/ 10.1055/s-0029-1215194

Online-Publikation: 8. 10. 2009

Akt Dermatol 2010; 36:

139-141 @ Georg Thieme

Verlag KG Stuttgart · New York ISSN 0340-2541

\section{Korrespondenzadresse} Dr. med. Regina Renner Klinik für Dermatologie, Venerologie und Allergologie, Universität Leipzig A. ö.R. Philipp-Rosenthal-Str. 23 04103 Leipzig regina.renner@ medizin.uni-leipzig.de

\section{Zusammenfassung \\ $\nabla$}

Röntgenstrahlen, Gamma-Strahlen, UV- und sichtbares Licht sind Teil der elektromagnetischen Strahlung, aber mit unterschiedlicher Energie. UV-Licht stellt u. a. für den Lupus erythematodes einen bekannten Triggerfaktor dar, dagegen wird Röntgen- oder $\gamma$-Strahlung nur sehr selten als Triggerfaktor zur Auslösung spezifischer Dermatosen beschrieben. Am Beispiel einer Patientin

\section{Einleitung \\ $\nabla$}

Sichtbares Licht $(380-780 \mathrm{~nm})$, ultraviolette Strahlen (UV; 1-380 nm), Röntgenstrahlung $(10 \mathrm{pm}-1 \mathrm{~nm})$ ebenso wie $\gamma$-Strahlung (unter $0,15 \mathrm{~A})$ sind Teil der elektromagnetischen Strahlung, aber in ansteigender Energiedosis. UV-Licht ist ein bekannter Triggerfaktor für die Induktion eines Lupus erythematodes oder anderer dermatologischer Erkrankungen. Pathogenetisch wird angenommen, dass über eine Induktion der Apoptose durch UVB eine sowohl lokale und auch systemische Entzündungsreaktion und Exazerbation eines LE möglich ist [1-3]. Im Gegensatz dazu wird nur selten über die Provokation von spezifischen Dermatosen nach Röntgenoder $\gamma$-Strahlung berichtet. Wir berichten über eine Patientin mit subakut kutanem Lupus erythematodes (SCLE), die unter einer Ganzhirnradiatio eine ungewöhnliche Dermatitis entwickelte, die wir in Zusammenschau aller Befunde als Exazerbation des bekannten SCLE im Sinne eines Köbnerphänomens deuteten. mit bereits langjährig bekanntem subakut kutanem Lupus erythematodes (SCLE), die unter einer Radiatio aufgrund eines kleinzelligen Bronchialkarzinoms im Bestrahlungsareal des Kopfes eine ungewöhnliche Dermatitis entwickelte, sollen die verschiedenen Auslösefaktoren diskutiert werden. Möglich erscheint uns eine Exazerbation des bekannten SCLE im Sinne eines Köbnerphänomens durch elektromagnetische Bestrahlung jenseits ultravioletten Lichtes.

\section{Fallbericht \\ $\nabla$}

Anamnese und Anfangsbefunde

Bei der 50-jährigen Patientin war erstmalig 1995 im Alter von 38 Jahren ein SCLE aufgetreten. Erhöht nachweisbar waren die antinukleären Antikörper ANA 1 : 1280 (Normbereich < 1:160), antiHiston-AK 35,7 IU/ml (15-30), anti-SS-A/Ro-AK und SS-B/La-AK. Anti-ds-DNA-Ak schwankten im Krankheitsverlauf zwischen normwertig und grenzwertig erhöhten Werten. Der Photoprovokationstest zeigte eine erhöhte Lichtempfindlichkeit sowohl im UVA- als auch UVB-Spektrum. Formal erfüllte die Patientin jedoch nicht die ACRKriterien für einen systemischen Lupus erythematodes [4]. Von 1995 bis 2002 war sie mit Chloroquin $250 \mathrm{mg} / \mathrm{d}$ und seit $2002 \mathrm{mit}$ Hydroxychloroquin in Kombination mit $5 \mathrm{mg}$ Prednisolon/d behandelt worden. 2002 wurde eine diskrete Lungenfibrose im rechten Lungenlappen festgestellt mit aber nur leicht reduzierten Funktionsparametern. 2004 wurde dann überraschend ein kleinzelliges Bronchialkarzinom im rechten Lungenflügel diagnostiziert.

\section{Therapie und Verlauf}

Das Karzinom und die bei Erstdiagnose bereits vorhandenen mediastinalen Lymphknotenmetastasen wurden mit einem Zyklus Paclitaxel, Etoposid und Carboplatin behandelt, gefolgt von $5 \mathrm{Zy}$ - 
klen Carboplatin/Cisplatin plus Etoposid sowie einem Zyklus Topotecan mit nachfolgender Radiatio thorakal mit 6 und $15 \mathrm{MeV}$ Photonen in der 3D-CT-isozentrischen-6-Felder-Technik. Die Bestrahlungsserie dauerte 16 Tage mit 5 Sitzungen/Woche und jeweils $2.5 \mathrm{~Gy}$ (40 Gy gesamt). Diese Bestrahlungsserie wurde von der Patientin gut toleriert. Unter der initialen Chemotherapie war es allerdings zu einer Verschlechterung des bis dahin stabilen SCLE gekommen. Eine histologische Untersuchung der neu aufgetretenen Hautveränderungen (siehe $\bullet$ Abb. 1) bestätigte die klinische Verdachtsdiagnose SCLE. Spezifische Autoantikörper wie ANA, anti-SS-A/Ro-AK und SS-B/La-AK waren weiterhin nachweisbar.

In einer adjuvanten Behandlungssituation nach Chemotherapie und unter klinisch wieder kompletter Remission des exazerbierten SCLE wurde eine zusätzliche Ganzhirnradiatio in Maskenlagerung über eine opponierende asymmetrische isozentrische 2-Felder-Technik über 15 Tage an 5 Tagen/Woche mit jeweils 2 Gy (30 Gy gesamt) durchgeführt. Am Ende der Bestrahlungen entwickelte die Patientin eine massive Dermatitis im Bereich des bestrahlten Areals, welche wir anfangs als ungewöhnlich schwere und atypische Radiodermatitis interpretierten. Im Verlauf war aber aufgrund des zu diesem Zeitpunkt leicht triggerbaren SCLE und der ungewöhnlich heftigen und lang anhaltenden Manifestation auch eine Provokation des SCLE durch die Radiatio möglich. Es zeigte sich ein fleckiges, leicht infiltriertes Erythem mit diffuser Schuppung im bestrahlten Areal, welches über 3 Wochen persistierte $(\bullet$ Abb. 2$)$.

Unter einer Behandlung mit topischem Methylprednisolonaceponat verschwanden die Hautveränderungen innerhalb einiger Wochen. Aufgrund ihres stark reduzierten Gesundheitszustandes willigte die Patientin damals jedoch in keine weiteren Biopsien der betroffenen Hautregion ein.

Vitales Tumorgewebe konnte nach der onkologischen Behandlung durch apparative Untersuchungen nicht mehr nachgewiesen werden. Nach einer nur kurzen Zeit mit stabilem Befund kam es jedoch 2006 zu einem Rückfall des Tumorleidens mit generalisierten Metastasen in Lunge, den Lymphknoten, der Leber und des Mediastinums. Nach nochmals 4 Zyklen Doxetacel kam es erneut zu einer Exazerbation des SCLE mit klinisch eindeutigen Hautveränderungen am Integument, sodass sowohl systemische als auch topische Glukokortikosteroide zur Behandlung eingesetzt werden mussten. 2007 verstarb die Patientin in Folge ihres metastasierten Lungenkarzinoms nach insgesamt 3-jährigem Krankheitsverlauf.

\section{Diskussion}

\section{$\nabla$}

Die Provokation von Autoimmunerkrankungen oder anderen Dermatosen unter einer Radiotherapie wurde bisher nur selten beschrieben [5] und können initial als Radiodermatitis imponieren [6]. Die Provokation von Dermatosen wie Psoriasis oder LE werden als Ausdruck des Köbner-Phämonens diskutiert [7-10]. Das Köbner-Phänomen bringt erkrankungstypische Hautveränderungen in Zusammenhang mit physikalischen oder chemischen Stimuli, die sogenannten isomorphen Stimuli, sodass sich die Hautveränderungen hauptsächlich im Bereich deren Einwirkung zeigen. Pathogenetisch können nach Einwirkung elektromagnetischer Strahlung zwei wesentliche pathogenetische Schritte angenommen werden: Während eines ersten unspezifischen Schrittes werden inflammatorische Zytokine, Stressproteine, Adhäsionsmoleküle oder Autoantigene aus dem Intrazellulär-

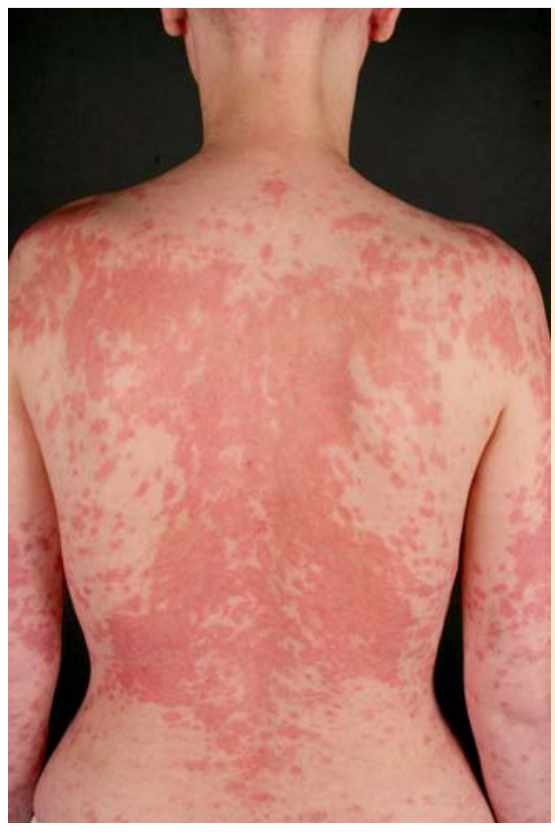

Abb. 1 Exazerbation des bekannten SCLE.

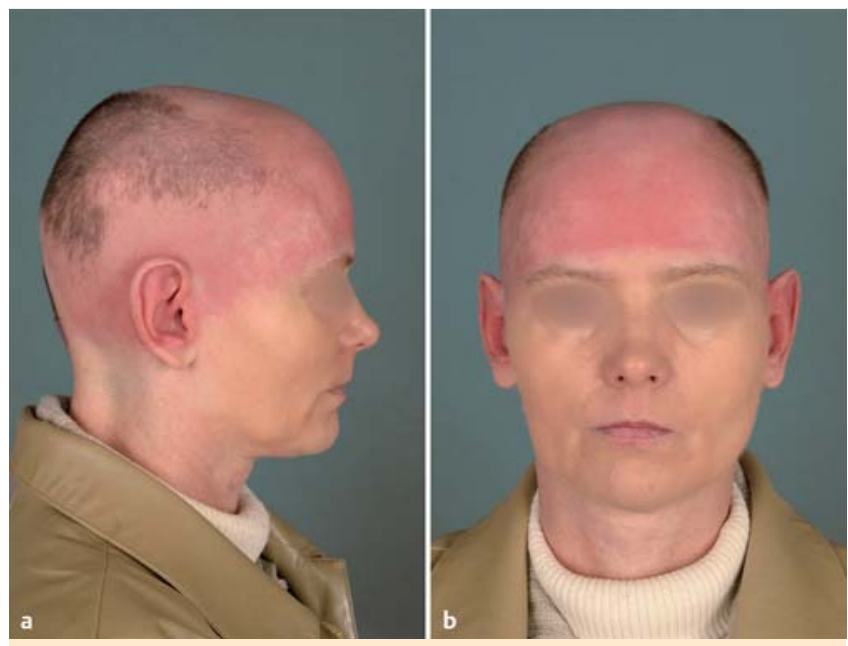

Abb. 2 a u. b Kopf der Patientin nach Ganzhirnradiatio mit ausgeprägter und lang persistierender Dermatitis.

raum freigesetzt. Nach einer Latenzperiode treten krankheitsspezifische Reaktionen hinzu, die durch im zweiten Schritt aktivierte T-Zellen, B-Zellen oder verschiedene Autoantikörper gesteuert werden [9]. UV-Licht und andere elektromagnetische Strahlung unterscheiden sich lediglich durch ihre Energie. Auch das sichtbar Licht entspricht einer elektromagnetischen Welle. Es stellt jedoch nur einen geringen Teil des gesamten elektromagnetischen Spektrums dar. Die kleinste Menge an elektromagnetischer Strahlung beliebiger Frequenz ist ein Photon. Bei niedrigeren Frequenzen ist die Energie der Photonen zu gering, um chemische Prozesse auslösen zu können. Bei höheren Frequenzen hingegen wirken Photonen auf Materie ionisierend.

Die DNA-Reparatur läuft nach Einwirkung ionisierender Strahlung bei Patienten mit Autoimmundermatosen verzögert ab. Zudem weisen Patienten mit Autoimmundermatosen einen signifikant höheren DNA-Schaden nach Bestrahlung auf im Vergleich zu gesunden Personen [11], was über eine erhöhte Apoptoserate die erhöhte Produktion von Autoantikörpern auslöst. 
Die erneute Exazerbation des bis dahin stabil eingestellten SCLE unter Chemotherapie in unserem Fall kam vermutlich aufgrund der Freisetzung von Autoantigenen infolge Nekrose/Apoptose zustande, die von einer Autoimmunreaktion gefolgt wurde. In Rückschau der Befunde mit zu diesem Zeitpunkt offensichtlich leicht triggerbarem SCLE und der ungewöhnlich heftigen und lang anhaltenden Manifestation der Dermatitis halten wir eine lokale Provokation des SCLE im Sinne eines Köbner-Phänomens durch die Radiatio für möglich. Problematisch bleibt jedoch die fehlende Sicherung der Diagnose mittels Hautbiopsie.

Bei einigen der verwendeten Chemotherapeutika wie Paclitaxel [12 -14], Doxetaxel [15] oder Cisplatin [16] sind zwar phototoxische Reaktionen beschrieben, aber ungewöhnlich. Paclitaxel wurde auch mit der Induktion eines SCLE in Verbindung gebracht $[17,18]$, ist aber ebenfalls sehr selten, sodass eine phototoxische oder medikamentöse $[18,19]$ Induktion der Hautveränderungen am Kapillitium bei unserer Patientin unwahrscheinlich ist, zumal Paclitaxel nur zu Beginn der Chemotherapien eingesetzt wurde. Die beginnende Verschlechterung des SCLE unter der Chemotherapie wäre jedoch zumindest partiell dadurch erklärbar.

\section{Fazit}

Die Radiotherapie besitzt ein moderates Risiko hinsichtlich einer akuten oder chronischen Toxizität bei Patienten mit Autoimmunerkrankungen, sodass diese keine Kontraindikationen für eine Radiotherapie darstellen [20]. Bei ungewöhnlicher Hautreaktion nach Radiatio sollte jedoch bei dieser Risikopopulation unbedingt an eine Köbnerisierung der Autoimmunerkrankung durch die ionisierenden Strahlen gedacht werden.

\section{Abstract}

\section{Radiation-Induced Subacute Cutaneous Lupus Erythematosus or Extraordinary Radiodermatitis? $\nabla$}

X-rays, gamma-radiation, UV and visible light are part of the electromagnetic radiation but in ascending energy dosage. UV light is a well known trigger factor e.g. for lupus erythematosus. In contrast $x$-rays and $\gamma$-radiation have only rarely been described or studied as provocating specific dermatoses. A patient with long-standing SCLE and metastasizing lung cancer is reported with atypical skin lesions at the area of cranial radiotherapy that may be interpreted in different ways. One possible explanation in our case might be exacerbation of SCLE because of the Koebner phenomenon induced by electromagnetic radiation apart from ultraviolet light.

\section{Literatur}

1 Schmidt E, Tony HP, Bröcker EB, Kneitz C. Sun-induced life-threatening lupus nephritis. Ann N Y Acad Sci 2007; 1108: 35-40

2 Bijl M, Kallenberg CGM. Ultraviolet light and cutaneous lupus. Lupus 2006; 15: 724-727

3 Meller S, Winterberg F, Gilliet $M$ et al. Ultraviolet radiation-induced injury, chemokines, and leukocyte recruitment. Arthitis Rheum 2005; 52: $1504-1516$

4 Ad hoc Committee Response Criteria For Cutaneous SLE. Response criteria for cutaneous SLE in clinical trials. Clin Exp Rheumatol 2007; 25: $666-671$

5 Carrote-Lefebvre I, Delaporte E, Mirabel X, Piette F. Complications cutanées de la radiothérapie (hors tumeurs malignes). Bull Cancer 2003; 90: $319-325$

6 Balabanova MB, Botev IN, Michailova JI. Subacute cutaneous lupus erythematosus induced by radiation therapy. Br J Dermatol 1997; 137: $648-649$

7 Charalambous H, Bloomfield D. Psoriasis and radiotherapy: exacerbation of psoriais following radiotherapy for carcinoma of the breast (the Koebner phenomenon). Clin Oncol (R Coll Radiol) 2000; 12: $192-193$

8 Schreiber GJ, Muller-Runkel R. Exacerbation of psoriasis after megavoltage irradiation. Cancer 1991; 67: 588-589

9 Ueki $\mathrm{H}$. Koebner phenomenon in lupus erythematosus with special consideration of clinical findings. Autoimmunity Reviews 2005; 4 : 219-223

10 Ueki H. Köbner Phänomen in Lupus erythematodes. Hautarzt 1994; 45: $154-160$

11 McCurdy D, Tai LQ Frias S, Wang Z. Delayed repair of DNA damage by ionizing radiation in cells from patients with juvenile systemic lupus erythemtatosus and rheumatoid arthritis. Radiat Res 1997; 147: 48 54

12 Cohen AD, Mermershtain W, Geffen DB et al. Cutaneous photosensitivity induced by paclitaxel and trastuzumab therapy associated with aberrations in the biosynthesis of porphyrins. J Dermatol Treat 2005; 16: $19-21$

13 Ee HL, Yosipovitch G. Photo recall phenomen: an adverse reaction to taxanes. Dermatology 2003; 207: 196-198

14 Cohen PR. Photodistributed erythema multiforme: paclitaxel-related, photosensitive conditions in patients with cancer. J Drugs Dermatol 2009; 8: $61-64$

15 Kaya AO, Buyukberber S, Coskun U et al. Acute erythema and edematous skin reaction and ectropion following doxetaxel in a patient with non-small cell lung cancer. Cutan Ocul Toxicol 2008; 27: 327-331

16 Nolè $F$, Munzone E, Mandalà $M$ et al. Vinorelbine, cisplatin and continuous infusion of 5-fluorouracil in metastatic breast cancer patients: a phase II study. Ann Oncol 2001; 12: 95-100

17 Adachi A, Horikawa T. Paclitaxel-induced cutaneous lupus erythematosus in patients with serum anti-SSA/Ro antibody. J Dermatol 2007; 34: $473-476$

18 Dasanu CA, Alexandrescu DT. Systemic lupus erythematosus associated with paclitaxel use in the treatment of ovarian cancer. South Med J 2008; 101: $1161-1162$

19 Sontheimer RD, Henderson CL, Grau RH. Drug-induced subacute cutaneous lupus erythematosus: a paradigm for bedside-to-bench patient oriented translation clinical investigation. Arch Dermatol Res 2009; 301: $65-70$

20 Pinn ME, Gold DG, Petersen IA et al. Systemic lupus erythematosus, radiotherapy, and the risk of acute and chronic toxicity: the Mayo Clinic Experience. Int J Radiat Oncol Biol Phys. 2008; 71: 498 -506 